\title{
Filigrane
}

Écoutes psychanalytiques

\section{Rêver pour comprendre. Entretien avec Vital Vézina}

\section{Réal Laperrière}

Volume 24, numéro 2, automne 2015

URI : https://id.erudit.org/iderudit/1036536ar

DOI : https://doi.org/10.7202/1036536ar

Aller au sommaire du numéro

\section{Éditeur(s)}

Revue Santé mentale au Québec

ISSN

1192-1412 (imprimé)

1911-4656 (numérique)

Découvrir la revue

Citer ce document

Laperrière, R. (2015). Rêver pour comprendre. Entretien avec Vital Vézina.

Filigrane, 24(2), 139-160. https://doi.org/10.7202/1036536ar d'utilisation que vous pouvez consulter en ligne.

https://apropos.erudit.org/fr/usagers/politique-dutilisation/ 


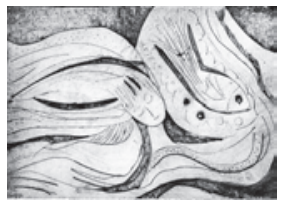

\section{Rêver pour comprendre. Entretien avec Vital Vézina}

\section{Réal Laperrière}

ital Vézina est docteur en psychologie et psychanalyste, membre de la Société psychanalytique de Montréal, de la Société canadienne de psychanalyse et de l'Association psychanalytique internationale. Il se définit avant tout comme un clinicien, et a cuvré auprès de clientèles diverses (patients psychiatriques, toxicomanes, alcooliques, enfants et adolescents), en suivis individuels ou de groupe. Après avoir travaillé en milieu hospitalier, il se consacre entièrement, depuis plus de vingt ans, à une pratique privée. Il a longtemps collaboré à un programme de formation à la psychothérapie psychanalytique, et a travaillé pendant plus de dix ans en séminaire sur la psychose, le narcissisme et le rêve. Nous l'avons rencontré dans son cabinet de consultation situé dans le quartier Côte-des-Neiges à Montréal.

Réal Laperrière: D’abord merci, Vital, de me recevoir dans ton lieu de travail, dans ton bureau de consultation. Je remarque que l'on est entouré d'œuvres d'art, de peintures sur les murs, de sculptures, de photographies... des photographies de fresques...

Vital Vézina: ... de la cathédrale d'Autun en Bourgogne.

RL: Je constate l'importance pour toi de l'art dont tu t'entoures dans ton bureau. Est-ce qu'au départ, si je me souviens bien, ta thèse de doctorat en psychologie ne portait pas sur les processus créateurs, notamment chez les peintres?

VV: Oui, plus précisément, l'étude des processus créateurs chez l'artiste et le psychothérapeute. J'ai utilisé le test de Rorschach auprès de trois populations: des psychothérapeutes d'orientation analytique, des peintres réputés du Québec et un groupe contrôle. Ce qui se dégage de cette étude, c'est que l'artiste et le psychothérapeute se rejoignent dans l'accessibilité à l'inconscient mais se différencient dans la qualité de ce processus. L'artiste a un accès plus profond, dans le sens plus primitif, comparé au psychothérapeute qui est plus contrôlé dans sa régression et maintient un meilleur contact avec la réalité. De plus, on a pu mettre en évidence que l'énergie à la base du travail 
artistique et du travail thérapeutique puisait son origine, dans les deux cas, au niveau de la composante orale de nature réceptive de la personnalité. Ceci renvoie au caractère réceptif de l'inspiration artistique et à l'ouverture empathique au patient chez le psychothérapeute.

RL: Donc il y avait vraiment une différence au niveau du rapport à l'inconscient chez les artistes?

VV: Oui, comme je disais, l'artiste est capable d'entrer en contact avec des contenus plus primitifs. Il est, pour ainsi dire, seul devant sa toile. Pour donner un exemple, à la planche trois du Rorschach, l'artiste peut dire, dans un premier temps: «des taches de sang, des corps en décomposition », mais dans un deuxième temps, il peut ajouter: "ça me fait penser à une scène de film, à un rêve, à une lecture que j'ai faite». On rejoint ici la notion de «régression au service du moi». Il peut faire quelque chose avec sa pulsion, comparé, si l'on veut, au psychotique qui lui est complètement envahi. Le psychothérapeute, par ailleurs, n'est jamais seul, le patient est là, il doit en tenir compte constamment. D'où l'importance d'un meilleur contact avec la réalité.

RL: Plus tard dans notre entretien, on reviendra à la question des processus créateurs, en lien avec le texte que tu as écrit sur Romain Gary. Mais je ne peux m'empêcher de poser cette question maintenant: peut-on dire que la réalisation d'œuvres chez un artiste (on parle des peintres mais on pourrait aussi parler des écrivains) est garante d'une vie créative au sens de Winnicott, une vie où il y a contact avec le self? Si on pense à Romain Gary, par exemple, est-ce qu'on pourrait penser que son œuvre lui a permis cette vie créative?

VV: En fait, la question que tu poses est exactement ce qui m'a amené à me pencher sur l'œuvre de Romain Gary. On sait qu'il se suicide en décembre 1980. Il est alors dans un état dépressif majeur; il a le sentiment d'être passé à côté de sa vie, d'avoir été un imposteur et pourtant, sur un plan social, extérieur, il offre l'exemple d'une vie réussie et créatrice.

RL: C'est plutôt un exemple de réussite.

VV: Du moins au niveau extérieur, social. Pour reprendre ta question, la réalisation d'œuvres créatrices n'est pas garante pour autant d'une vie réussie au niveau affectif. Il s'agit de comprendre la place que prend la création, dans l'aménagement économique du sujet. Chez Gary, comme je le mentionne, la création lui permettait de maintenir un sentiment d'identité. Il disait lui-même que les femmes et l'écriture lui avaient permis de vivre. Son écriture reposait sur une base traumatique comme je le démontre.

RL: On reviendra tout à l'heure à ce texte sur Gary, qui est plus récent que les autres dans le parcours de tes écrits. Mais revenons-en au départ: je me 
demandais ce qui t'a amené à choisir la psychologie et ensuite la psychanalyse? Qu'est-ce que tu peux en dire aujourd'hui?

VV: Je dois dire que la psychanalyse a toujours été présente, elle est entrée dans ma vie vers l'âge de 13-14 ans. Je lis à ce moment-là Les triomphes de la Psychanalyse et Les prodigieuses victoires de la psychologie moderne de Pierre Daco et pour moi, ce fut un moment majeur dans ma vie, un moment fondateur, si je peux dire. Le livre était basé sur des exemples de comportements: monsieur $\mathrm{X}$ arrive, il est anxieux, ou il a peur, ou n'arrive pas à dormir, etc. Dans un deuxième temps, il explique un peu la dynamique, ce qui se passe à l'intérieur de la personne et qui est relié à son histoire. Alors pour moi, ce fut une révélation. Je me sentais mal à ce moment-là, je me sentais angoissé, j'avais l'impression de vivre avec un fardeau qui serait toujours là. Je comprends alors que ce que je ressens a du sens, il y a une explication possible. Il s'agit d'aller voir et c'est là que mon désir est né. Pour moi c'était clair, à 14 ans, que je deviendrais psychanalyste. Mais c'était surtout clair que je me retrouverais sur un divan à un moment donné, il s'agissait de tenir le coup. Donc, mes études, mon parcours ont fait qu'à un moment donné, j'ai pu. RL: Le désir d'analyse personnelle a donc précédé le choix de la psychologie? VV: Oui. Pour moi, à un moment donné, je trouverais quelqu'un qui m'aiderait (aujourd'hui je peux le dire) à me penser. C'est le besoin de comprendre qui m’a amené vers l'analyse.

RL: Et donc la psychologie en était le chemin?

VV: Ce n'était pas si clair. Je viens d'une petite région et d'une époque où on ne parlait pas d'analyse, ni d'analystes. Donc, pour moi, psychologie et psychanalyse c'était équivalent, je ne voyais pas la différence. C'est peu à peu que tout ça a pris sens.

RL: J'imagine qu'à ce moment-là, les études en psychologie étaient plus imprégnées de psychanalyse que maintenant.

VV: Bien sûr!

RL: On reviendra aussi plus spécifiquement à ton parcours comme analyste, et aux rencontres qui ont été marquantes. Mais j'aimerais maintenant que l'on parle de tes écrits, que j'ai relus pour l'entretien. Le premier texte que tu as écrit est paru en 1985 et portait sur le psychodrame, plus précisément sur une expérience de psychodrame analytique avec des enfants à l'hôpital Notre-Dame. Mais tout d'abord, qu'est-ce qui t'a amené à vouloir travailler avec les enfants?

VV: En fait, disons que, à la base de mes motivations, le côté créateur, spontané, ludique de l'enfant me rejoignait et, aussi, j'avais l'impression (même si 
j'ai changé d'idée depuis) que le travail avec un enfant serait plus facile, plus rapide, que l'enfant était moins blindé au niveau des défenses. Rapidement, je me suis rendu compte de ma méprise: que tu aies 30 ans ou que tu en aies 8 , ta carapace est quasiment aussi importante. Il y avait aussi, par rapport au psychodrame, un vieux rêve de devenir comédien. Enfant, je faisais des pièces de théâtre, je montais des spectacles, des tombolas, des parades. Je dois dire que, assez tôt, j'étais fasciné par le fait de créer, d'inventer. Je me couchais le soir avec des idées plein la tête que je pourrais élaborer le lendemain matin. Puis, c'est au niveau du baccalauréat en psychologie que j'ai pris contact avec le psychodrame de Moreno. Par la suite, j'ai découvert Anzieu et son approche analytique du psychodrame, ce qui me rejoignait davantage, l'approche de Moreno étant plus comportementale. J'ai fait une première année de formation en psychodrame à Louvain en Belgique et ensuite, j'ai complété ma formation ici, à Montréal, à l'institut mis sur pied par Carmen Des Francs.

RL: Carmen Des Francs qui travaillait alors comme psychologue à l'hôpital Notre-Dame.

VV: Oui. Je l'avais eue comme superviseure au niveau du doctorat, à l'hôpital Notre-Dame. Par la suite, je fus intégré au département de pédopsychiatrie. J'y dirigeai des groupes de psychodrame avec les enfants pendant 11 ans. Certaines années, je faisais trois groupes/semaine. Je trouvais que c'était un excellent outil thérapeutique et de plus, c'était économiquement rentable: sept enfants par groupe avec deux thérapeutes. Les enfants qui consultaient à l'hôpital Notre-Dame répondaient mal à la thérapie individuelle. Ils ne jouaient pas, ils paralysaient dans la relation duelle, alors que le groupe correspondait plus à ce qu'ils connaissaient, c'était un espace beaucoup plus accessible pour eux.

RL: Oui, ce que tu dis dans le texte, c'est que pour les enfants qui ont des problèmes de mentalisation, le psychodrame de groupe est particulièrement indiqué...

VV: Oui, exactement.

RL: Et en pédopsychiatrie, il s'agit d'une bonne partie de la clientèle... VV: Surtout à l'hôpital Notre-Dame.

RL: Tu trouvais donc que c'était plus fructueux comme travail que le suivi individuel. Mais en même temps, tu décris bien la «folie» de ces groupeslà. C'est angoissant en fait. À un moment donné tu racontes une séance et tu expliques: «Nous nous sentons démunis et impuissants, incapables de trouver les bonnes interprétations, incapables même de penser. C’est la 
discontinuité, la rupture, le chaos.» Je pense que tous ceux qui ont travaillé avec des groupes d'enfants dans un contexte peu directif ont vécu cela. Il faut donc faire quelque chose avec ce chaos. Lorsque tu expliques que le groupe est ce qu'il y a de plus acceptable pour le self, c'est paradoxal, parce qu'à te lire, l'on voit bien que c'est très angoissant pour les enfants. Plusieurs autres l'ont constaté auparavant. Bion a discuté des angoisses psychotiques qui sont soulevées par la mise en groupe. Mais en même temps, c'est le dispositif qui permet le mieux à ces enfants de progresser.

VV: Oui, j'ai vraiment saisi toute l'importance du tiers, l'importance de la loi du père, si on veut. Il fallait beaucoup de courage quand je repense à ça! Après quelques semaines, on ne pouvait plus continuer le groupe, c'était le free-for-all: les enfants grimpaient dans les fenêtres, se battaient, sortaient dans la clinique, se mettaient à hurler. Il y avait un groupe de parents qui était, au même moment, dans une autre salle. Les enfants allaient frapper dans la porte, menaçaient de défoncer... Les parents ne savaient plus quoi faire, leur groupe était mis en morceaux. C'est là que m'est tombé entre les mains le livre de Gérard Decherf, Edipe en groupe, ce qui a donné du sens à ce que je vivais dans le groupe. Nous avons donc réajusté notre manière d'être avec les enfants. C'était devenu tolérance zéro: les enfants arrivaient et s'ils commençaient à déconner, on terminait le groupe. Le groupe pouvait, surtout au début, ne durer que cinq minutes. Les enfants étaient avertis qu'on ne tolèrerait aucun écart de conduite. Paradoxalement, ils sortaient très calmes, ils allaient s'asseoir dans la salle d'attente et lisaient, attendant que le groupe de parents se termine. De semaine en semaine, le même phénomène s'est reproduit. Peu à peu, ils sont restés de plus en plus longtemps et un vrai processus de groupe s'est mis en place, un vrai processus de représentation, de symbolisation où les enfants ont repris, à travers des thèmes mais sous forme de fantasmes, des attaques de dragons, de serpents, d'ours. Tout ce qui avait été agi dans la première période, était maintenant représenté... Donc, à partir de ce moment, nous avons pu analyser.

RL: Mais, tu dis bien aussi, par ailleurs, qu'il n'y avait pas d'interprétations individuelles.

VV: Non, c'est au niveau du groupe. C'était un groupe de sept, donc il pouvait $y$ avoir deux ou trois thèmes proposés par les enfants. Le thème qui obtenait le plus de votes était joué. On pouvait supposer que ce thème représentait la dynamique qui circulait dans le groupe à ce moment-là. Lorsque le thème était joué, on «le parlait» en termes de groupe: le groupe présentement est inquiet, le groupe se questionne, le groupe est agressif. Ces 
interprétations de groupe sont reçues de façon moins envahissante par l'enfant, ce qui constitue l'approche d'Anzieu.

RL: Justement, tu expliques aussi que dans l'évolution du groupe, un moment important est l'accès au «on» qui relève de l'illusion groupale décrite par Didier Anzieu. Celui-ci y voit une défense contre l'angoisse persécutrice vécue par la mise en groupe. Le «on» est aussi un étayage à l'identité groupale. Je trouve intéressante la façon dont tu formules dans le texte que le "on", c'est du moi et du pas moi en même temps.

VV: C'est du transitionnel.

RL: Oui, je ne l'avais pas pensé comme cela; l'illusion groupale relève donc pour toi de la transitionnalité.

VV: Oui.

RL: Le groupe serait donc rentable, non seulement au plan clinique parce que parfois mieux adapté que le cadre individuel, mais aussi au niveau économique parce que deux thérapeutes travaillent avec six ou sept patients simultanément? Pourtant, il n'y a pas beaucoup de cliniciens au Québec qui travaillent avec des groupes, alors qu'en France, c'est depuis longtemps bien établi. Pourquoi est-ce qu'ici les thérapies de groupe ne sont pas installées comme pratiques cliniques en institution?

VV: Je ne sais vraiment pas, car effectivement, c'est un outil très rentable à tous points de vue.

RL: Je me demande si, chez les analystes québécois, il n’y a pas une résistance à envisager des pratiques de groupe.

VV: Effectivement, je pense qu'on peut, à tout le moins, parler d'un manque d'intérêt.

RL: Je me suis demandé si les analystes n'avaient pas un peu peur de perdre leur identité en se prêtant à des pratiques comme le psychodrame...

VV: Oui probablement. Comme s'il fallait rester dans la cure type? Peutêtre est-ce dû au nombre plus restreint d'analystes?

RL: Puisqu'on parle des analystes, il y a un autre texte que tu as publié dans le Bulletin de la $S P M^{1}$, qui est un commentaire à un cas clinique exposé par une collègue lors d'une réunion scientifique à la Société. Tu y avais introduit l'idée que chez certains patients, il y ait un self omnipotent qui se pose en ennemi de l'analyste. Cela se manifeste notamment par l'espèce de retenue, de timidité à interpréter. Comme si ce self nous amenait à retenir l'interprétation qui en même temps constitue la seule arme contre lui. À ce propos, je me demande comment tu conçois l'interprétation? Dans ce texte, tu expliques qu'elle a d'abord une fonction contenante, puis dans un 
deuxième temps, la fonction de fournir des contenus. Est-ce encore ainsi que tu conçois l'interprétation?

VV: Oui. Disons que ce qui m'apparaît central dans notre travail (et je dirais de plus en plus), c'est la place donnée au patient à l'intérieur de soi. Le patient doit sentir qu'il existe pour l'analyste. C'est là le premier contenant, ce qui lui donne un sentiment d'existence. D'ailleurs, les recherches en psychothérapie soulignent que le premier facteur d'amélioration est l'investissement de la psyché du sujet, de ce sujet-là par le clinicien. Comme le souligne A. Ferro: "l'unisson (dans le sens d'accordage) est la base pour qu'il y ait tout le reste». En psychanalyse, il y a la cure type, c'est important qu'il y ait des balises, mais je pense qu'il y a des aménagements qui doivent être faits pour chaque patient. Ces aménagements marquent la spécificité de la relation, créent l'unicité de celle-ci et font que le patient se sent contenu. Dans ce contexte, l'interprétation m'apparaît davantage comme le résultat d'une association au discours du patient. Je te donne un exemple:

Une femme est plutôt silencieuse dans une séance, elle parle peu. L'atmosphère est assez lourde. Dans ce silence, mes pensées vagabondent et se fixent sur les images d'un reportage que j'ai vu à la télé, quelques jours auparavant, sur la solitude des gens au Japon: on a créé des cafés où on laisse circuler des chats afin que les personnes puissent venir les caresser. Je lui parle de ce reportage. Elle se met à associer sur les différents petits animaux qu'elle a eus, enfant. Elle me parle d'un petit hamster qu'elle avait réussi à apprivoiser, me décrivant, avec détails, comment il venait sur son bras, grimpait dans son cou et se retrouvait sur sa tête blotti sur un chapeau de laine qu'elle portait pour l'occasion. Un soir, en hiver, rentrant à la maison, elle ne retrouve plus son hamster. Elle cherche partout et le retrouve à l'extérieur, mort, complètement gelé. Il s'était soustrait à une surveillance. «Ce fut un chagrin énorme, c'était ma première perte, j'ai mis longtemps à calmer cette peine». Pendant tout le temps qu'elle parle j'ai en tête l'image d'une petite fille de 6-7 ans et j'apprends alors qu'elle avait 17 ans! Je sens alors toute sa fragilité, sa vulnérabilité, et me vient l'image d'un oiseau, en hiver, qui essaie de se réchauffer. La rencontre se termine. Elle revient le lendemain avec deux images qui lui restent d'un rêve: un oiseau sur une branche balloté par le vent, et une autre image où elle doit fuir l'état islamique. Ceci nous a permis d'aborder d'un côté, son besoin de rapprochement et de l'autre, sa crainte de l'emprise. Ceci a aussi permis de mieux comprendre les grands moments de silence 
rencontrés dans le déroulement de la cure. C'est dans ce sens que je parle d'associations-interprétations.

RL: Toi, tu lui parles de ça comme une association venant de toi? VV: Oui c'est ça! Je fais de plus en plus confiance à cette rêverie, ce n'est pas pour rien (et cela rejoint Ogden) que je pense à ça, à ce moment-là, avec ce patient-là. C'est le véritable travail de liaison qui se fait au plus près de la pensée du patient. Je suis tout à fait d'accord lorsque Green mentionne que les interprétations profondes «matraquantes» comme il dit, n'ont que le pouvoir de renforcer le clivage.

RL: Ce que tu es en train de décrire, on va y venir parce que tu l'as développé dans deux de tes textes. Notamment, dans le texte écrit en 1995: Paradoxe, emprise et rupture. Ce texte se fonde sur le travail avec une patiente qui était une femme médecin dans la quarantaine, chez qui les frontières étaient fragiles, les limites mal assurées. C'est une patiente qui se présente à toi, à la première rencontre, dans un état de désespoir, avec une menace de suicide formulée dès la première séance, je pense. «Vous me prenez ou je me jette devant un wagon de métro en sortant d'ici». Disons que ça donne le ton! VV: Oui, ça a donné le ton!

RL: Tu écris comment cette patiente est incapable d'intimité parce qu'elle vit toujours les relations comme une effraction, mais en même temps, elle fait vivre cela à l'autre aussi. Évidemment, dès la première séance, il y a un effet d'effraction chez toi. Tu dis comment chez elle, et là on va venir à ce que tu avais commencé à élaborer, il y a la recherche d'un espace de croissance, comme un moteur dans sa vie, mais ça ne marche pas. L'espace, elle le recherche dans les voyages, dans l'alcool qui lui occasionne des problèmes sérieux, de même que dans son travail auprès des enfants mourants. Elle s'est un peu spécialisée là-dedans. Là où on en revient à ce que tu avais commencé à élaborer, c'est lorsque tu dis que ce qui a donné cet espace-là à cette patiente, c'est l'aménagement du cadre. Quand on te lit, on pense d'abord à l'aménagement du dispositif mais tu dis, si j'ai bien compris, qu'avant que ce soit un aménagement de dispositif, ça doit être un aménagement interne, c'est-à-dire un espace donné au patient à l'intérieur de l'analyste. Que c'est cet aménagement-là qui est le plus fondateur et que c'est le premier contenant, c'est un peu ça que tu dis?

VV: Oui, c'est ça, exactement.

RL: Et c'est là que tu parles du recours à la rêverie. Comme dans l'exemple que tu as donné juste avant avec ton patient et le Japon... 
VV: Oui, c'est ça. En fait, c'est plutôt dans un autre texte que j'ai élaboré cette notion de rêverie.

RL: Oui, dans l'autre texte qui suit tu en donnes un exemple frappant, on va y venir. Mais pour en rester à cette patiente, tu écris: «à un moment donné je comprends que je m'adresse à quelqu'un qui n'existe pas». Ça apparait comme un moment très important lorsque tu réalises cela. On comprend qu'il n'y a pas chez elle de lieu pour recevoir ce que tu lui dis.

VV: Exactement! Il n'y a pas de pensée, pas d'élaboration, pas de lieu interne. On peut dire qu'on est dans l'actuel. Il faut arriver à le lui donner, à créer un espace pour qu'elle puisse arriver à penser. Donc, avant même que l'analyse commence, si on peut dire cela comme ça, il y a tout un travail à faire d'aménagement d'un appareil à penser. La manière d'être de l'analyste dans cette situation rejoint ce que Green propose lorsqu'il mentionne que la réponse du psychanalyste doit être celle que le patient n'a pas reçue des objets primaires. Ainsi, avant tout, avant qu'il n'y ait un processus de pensée, de liaison, ou en fait une analyse, il faut qu'il y ait un espace qui soit créé et ce premier espace est vraiment à l'intérieur, dans l'analyste, dans sa psyché.

RL: En même temps tu dis que et je te cite: «Ce processus d'identification au monde interne du patient est peut-être la tâche la plus ardue». Pourquoi donc?

VV: C'est-à-dire qu'il y a un certain abandon (processus que j'ai pu identifier, du reste, dans la recherche dont on a parlé plus haut); il faut que tu te laisses aller, que tu te laisses pénétrer par le patient, par son monde interne. On rejoint des fantasmes très primaires et ce n'est pas facile de les assumer, d'avoir ces fantasmes, de vivre ces mouvements de haine, de rage et, en même temps, d'être capable de les contenir. Sinon, on peut agir dans la relation d'une façon ou d'une autre, soit en finissant la rencontre plus vite ou en oubliant la patiente. Face à tout cela, on a besoin d'être bien contenu comme analyste.

RL: Cette patiente avait chez elle toute une problématique, si on pense à «La mère morte» de Green. Il y avait quelque chose de cet ordre-là parce que tu expliques que c'est une patiente qui avait eu elle-même une mère endeuillée, une mère dépressive et qu'elle l'avait semble-t-il incorporée. Elle était devenue son cercueil, d'une certaine façon. Pour que les deux survivent, tu supposes qu'elle avait fait «don de son self à l'objet». Selon toi, son self était écrasé entre l'objet et son faux-self, sans possibilité de croissance. Ce qui avait comme résultat que la vie était ressentie, par la patiente, comme une 
trahison face à l'objet, autrement dit: «si je vis, je tue l'objet». Vivre était en soi culpabilisant. Alors, il fallait la sortir du cercueil, si je comprends bien. VV: Oui. Il y a eu une représentation assez éloquente à ce sujet. À la seconde rencontre, elle vient et me parle d'un rêve qu'elle a fait durant la semaine: c'était un homme qui brisait une espèce de bunker en béton. C'était l'espoir qu'elle avait que j'arrive finalement à la sortir de cette espèce de cercueil, de tombeau dans lequel elle était enfermée.

RL: Tu insistes dans le texte sur la nécessité d'une attitude de support. Soutenir les élans créateurs du self. Par exemple on peut penser que dans ce rêve-là, qui semble apparaitre en début de traitement, il y avait comme une manifestation d'un self qui veut exister. Tu parles de l'attitude de support, mais tu sais bien que support n'est pas un terme très prisé par les analystes; alors pour toi ça va jusqu'où? Qu'est-ce que tu entends par le support?

VV: Je dirais supporter les élans de vie d'un self en développement. Offrir un support aux manifestations de désirs qui peuvent commencer à s'exprimer, analyser les résistances. Par exemple, comme je décris cette patiente au début, il y avait vraiment une abdication chez elle: pas de pulsionnel du tout, pas de bijou, pas de maquillage, rien, vraiment une religieuse, une vie sacrifiée. Donc, elle avait vu un collier, à un moment donné, et me faisait part de ses réticences: «Ah! Je ne sais pas! C’est cher! Je n’ai jamais mis ça.» «Bon, alors pourquoi pas?» Au sens de: «Pourquoi ne l'achèteriez-vous pas ce collier? Pourquoi n'iriez- vous pas à cette soirée?»

RL: Donc, c'est un support au self qui pourrait se permettre de...?

VV: Pour essayer de neutraliser le plus possible cet espèce de surmoi très sadique, très tyrannique.

RL: Qui se manifeste par une culpabilité à exister?

VV: C'est ça! Tu sais, j'ai l'impression que c'est un des problèmes centraux de notre travail: amener le patient à se désidentifier de la mère. Ce n'est pas évident! La culpabilité est énorme à avoir une vie différente, meilleure; bref, une vie à soi, car elle repose sur une colère souvent impensable, irreprésentable. Green, d'ailleurs, dans un de ses derniers ouvrages, mentionne que souvent, la haine à l'égard de la mère n'admet aucune réconciliation et demeure fixée. Il dit quelque chose comme: «La mère primitive reste inoubliable.» J'ai de nombreux exemples, dans ma clinique, qui corroborent ces dires. Actuellement, je travaille cette dimension avec un patient et ça me trouble de constater la puissance de ce mécanisme: dans une rencontre, il va ressentir un certain bien-être, va avoir des projets, se sentir plus vivant et le lendemain immanquablement, se sent paniqué, étourdi, angoissé et une 
difficulté énorme à faire sa journée. "Elle s'acharne contre nous» dira-t-il. Ce patient est allé, si je peux dire, jusqu'à la crise cardiaque! Je trouve ça très troublant. J'ai vécu quelque chose de semblable avec une patiente morte du même cancer et au même âge que sa mère. Ses rêves représentaient une rage et une colère énorme, mais elle restait incapable de se l'approprier: "Elle m'a quand même aimée!» «Ce n'est pas possible d'avoir autant de colère!» Mes efforts pour rendre la colère plus acceptable — en parlant de la colère de l'enfant qui est totale, sans nuances - restaient vains. Il y avait une fermeture complète. Je ne vais pas jusqu'à dire que ça aurait pu la sauver, mais ça l'aurait certainement aidée.

RL: On ne peut pas toujours empêcher cette force-là...

VV: Eh non! C'est là que la force de Thanatos prend tout son sens. Ce fut un épisode difficile à vivre pour moi. Ça m’a amené à un travail introspectif important: est-ce que j'ai été sourd à une certaine dimension? Et la colère par rapport à ma propre mère? Je pense que souvent on peut, sans en être conscient, avoir une écoute très sélective et se fermer à des pans importants de la psyché de l'autre.

RL: Parlons maintenant de ce texte paru en 2004, Un lion dans la jungle, qui est assez saisissant. Avant de présenter un cas clinique, tu évoques la notion de traumatisme surtout à partir de Freud et de ses derniers textes, L'homme Moïse et la religion monothéiste et Construction en analyse. Tu expliques comment le traumatisme reste enfoui au fond de l'inconscient et qu'il s'agit de trouver une façon de le faire apparaître dans la relation analytique puisqu'il est constamment remis en scène. Tu vas proposer que l'inconscient de l'analyste soit l'outil qui puisse permettre de le capter, de le rendre réel à nouveau par le contre-transfert. Tu fais un parcours historique de la notion de contretransfert pour en arriver à une définition plus contemporaine. Ça, c'est la partie théorique qui est fort bien développée, mais il y a le cas clinique d'une jeune femme de 25 ans qui s'appelle Louise. Ce qui est assez marquant dans cet exemple, c'est d'abord la première période de cette analyse. Ce que tu décris c'est qu'au début, tu fais avec elle une analyse très classique. C'est une névrose hystérique assez caractéristique, avec problématique odipienne, désir pour le père, rivalité avec la mère, culpabilité. Un beau cas-contrôle, quoi! Tout est là, on sait dans quoi on est.

VV: Je dirais même qu'on le sait trop bien! J'ai beaucoup appris avec cette patiente. Elle a été à l'origine de mon questionnement clinique, de la construction de ma théorie; bref, elle m'a beaucoup apporté. Comme je le mentionne dans ce texte, j'ai pu réajuster le tir en me fiant à ce que je 
ressentais dans mon corps, si je peux dire. En cela la psychanalyse m'apparaît, de plus en plus, comme un processus éminemment corporel. Puis, pour nourrir ma pensée, encore une fois, un livre est venu à ma rescousse: Le drame de l'enfant doué, dans lequel Alice Miller dénonce cette connivence qui peut s'établir entre patient et thérapeute: le patient doué va se conformer aux attentes de son thérapeute comme il l'a fait autrefois avec ses parents. Ainsi, tout a l'air de bien se dérouler, le patient associe, rêve, pleure, mais aucun processus ne prend forme. Pour reprendre les mots de Winnicott: «il n’y a personne». Le patient est complètement clivé, il ne fait que répondre à une demande. D'où l'importance de donner de la place à ce qu'on peut ressentir en présence du patient, surtout corporellement: fatigue, lassitude, engourdissement, vertiges, etc., et aussi les rêveries qui peuvent venir sous forme d'images, de fantasmes. Ce sont autant de manifestations qui nous aident à bien identifier ce qui se passe, dans la séance à ce moment-là.

RL: Oui! Tu décris dans le texte ta prise de conscience d'un lieu où tu étais avec elle. Tu dis que cette patiente-là te donnait du matériel névrotique, un peu comme un appât. Un peu comme «je te donne cela à manger et puis...» VV: Exactement: «Et puis, laisse-moi tranquille!»

RL: Cela lui permettait de se cacher parce qu'elle était terrorisée. C'est ça qui est assez saisissant dans la façon dont tu décris ce cas. À un moment donné tu décris une scène, je pense que c'est lorsqu'elle arrive avec ses sacs d'épicerie...

VV: C'est ça! J'avais mon bureau en face d'un supermarché à ce moment-là. RL: Puis, elle dépose ses sacs par terre et là, il y a de la nourriture qui roule sur le plancher du bureau. Il se passe alors quelque chose en toi, tu as l'impression de prendre contact avec des zones beaucoup plus archaïques.

VV: C'est comme un film qui se présente à moi. Pendant un certain temps, au moment de lui faire une interprétation, j'avais le fantasme de lui présenter, comme on fait avec les bébés, une cuillerée de céréales et qu'elle la repoussait en la faisant revoler. Je vois ça, je vois cette nourriture... une pomme qui roule sur le tapis, un contenant de jus, et je me dis: «Elle a amené sa bouffe avec elle.» Et c'est là que me vient cette perception d'un lion; je me dis: "C'est ça, elle est terrorisée.»

RL: Elle est terrorisée par ta présence?

VV: C'est ça!

RL: Mais tu dis aussi que c'est elle le nourrisson affamé, enragé.

VV: C'est ça: le lion!

RL: Qu'elle met en toi, par identification projective? 
VV: Plus tard, j'ai appris (elle ne me l'avait pas dit), qu'elle avait été un bébé anorexique dans les premiers mois; on avait dû recourir au gavage pour la tenir en vie. J'étais le bébé affamé prêt à sauter sur le sein et le détruire, et c'était contre ça qu'elle se défendait. Je me suis mis à lui parler tout simplement sans interprétation, rien.

RL: Lorsque tu dis qu'il faut que l'objet-analyste commence à exister dans la réalité, c'est ça que tu veux dire?

VV: Oui. Situer l'analyste en dehors des phénomènes de projection avant de pouvoir l'utiliser, comme le mentionne Winnicott.

RL: Pour l'analyste, à ce moment, il n'est pas question d'une position de retrait, de silence.

VV: Non, c'est ça. Donc, à ce moment, elle a commencé à me parler et je la questionnais sur ses activités de fin de semaine, les films qu'elle avait vus, ses rencontres avec les amis, etc. L'important était de lui dire où j'étais, de devenir plus réel, me disant: «Elle va savoir où je suis, elle n'aura pas à se cacher.» L'apprivoiser peu à peu. Ce que j'ai trouvé superbe est que six mois après, elle me dit exactement ce que j'avais, moi, fantasmé: «Je vous imagine comme un lion, etc.», et elle trouvait cela complètement fou. C'est ça que je veux dire: l'interprétation, la rêverie...

RL: Je pense que tu as lu le plus récent livre de Laurence Kahn: L'analyste apathique et le patient post-moderne. Elle ne va pas du tout dans le même sens que toi. Elle écrit à un moment donné: «la froideur de l'analyste, son abstinence et le refus de tout adoucissement sont les conditions sine qua non de la cure, c'est par cela que peut s'ouvrir la voie du fantasme et de la réalité psychique». On est ailleurs! Elle serait très critique de ta façon de travailler! VV: Complètement, c'est certain... Vois-tu, je reprends ce que Roussillon dit: le moi ne peut se construire qu'en manipulant l'objet, le médium malléable. Donc, il faut que l'objet-analyste se montre disponible, ouvert, vivant; pour moi c'est la condition sine qua non de la cure. Je pense qu'il n'y a pas qu'une seule manière d'être présent au patient: certains ont besoin qu'on reste silencieux, à distance, d'autres qu'on soit beaucoup plus présent, plus actif. Il s'agit de trouver le bon accordage, pour reprendre les paroles de la petite «Piggle» qui avait créé «son Winnicott»; il s'agit de créer son psychanalyste à soi, ce qui se fait à deux. J'ai reçu à l'occasion des patients pour une deuxième, voire une troisième «tranche». Chacun, avec des mots différents, exprimait la même déception, si je peux dire, de s'être buté au silence, à la froideur et d'en sortir en disant: "Ce n'est pas possible que ça ne soit que ça.» Bref, des analyses où il n'y avait pas eu de rencontre. Par exemple, quand 
un patient a eu une mère déprimée, silencieuse, une mère morte... il a besoin d'un psychanalyste vivant, sinon on n'avancera à rien, le trauma se répète, sans espace d'élaboration.

RL: Tu es beaucoup plus près de Roussillon que de Laurence Kahn, du moins dans son dernier livre...

VV: Oui, nettement.

RL: Alors, dans la façon dont tu travailles, l'analyste doit accepter de prêter au patient ses propres capacités de rêverie, d'éprouver ses pensées pour qu'il arrive à sentir ce que le patient n'a pas senti, qu'il éprouve ce qu'il n'a pas éprouvé, qu'il pense ce qu'il n'a pas pensé. Mais si je me fais l'avocat du diable: est-ce qu'il n'y aurait pas un danger que l'analyste impose au patient une vision de ce dernier qui serait modelée sur lui, qui serait construite sur ses propres éprouvés, son vécu, et que le patient reproduise en quelque sorte une aliénation qui aurait pu être vécue avant?

VV: Oui, c'est certain le danger peut être là: en voulant éviter l'aliénation, on en produit une. L'ouverture, la sensibilité, l'écoute m'apparaissent comme des paramètres essentiels qui peuvent nous guider avec sécurité dans notre travail. A ce propos, je pense à un livre de A. Ferro, Psychanalystes en supervision, qui m'a beaucoup aidé dans ce cheminement. Encore une fois, je le répète, la psychanalyse demeure un processus hautement corporel.

RL: On pourrait en venir maintenant à ton dernier texte qui portait sur Romain Gary. En fait, c'est d'abord un texte sur le rêve où tu reprends le parcours de ce qu'on en a compris à partir de Freud dans Rêves et réalisation de désir. Mais après ça, tu vas à la conception du rêve non comme réalisation de désir mais plutôt comme figuration du monde interne en t'inspirant de Klein, Bion et Meltzer qui ont beaucoup écrit là-dessus. Tu parles du rêve comme d'un outil indispensable au processus d'appropriation subjective. Je pensais quand même et je ne sais pas ce que tu aurais à en dire, à ces patients qu'on voit en clinique qui rêvent et même parfois rêvent beaucoup, qui nous en parlent en séance mais qui n'arrivent pas à en retirer quelque chose... VV:... le rêve qui n'est pas investi. Le rêve n'est pas utilisable... RL: Oui. Un peu comme le dirait Pontalis: «Ils ne font pas l'expérience du rêve.» Et même, on a l'impression parfois qu'ils n'arrivent pas à voir le rêve comme un rêve, comme une expérience intérieure qui peut les informer sur eux-mêmes, sur ce qui se passe en eux.

VV: J'ai eu de ces patients qui faisaient ce que j'appelle des rêves acting, des rêves acting out, au fond. Les rêves sont là, mais tu ne peux rien en faire, ils ne s'y arrêtent pas et pourtant, le contenu du rêve est intéressant. Mais il n'est 
pas investi, c'est comme une fuite. J'aime bien la formule de Pontalis dont tu parles: «Ils ne font pas l'expérience du rêve.» Comme si le patient disait: «Tu veux des rêves, je vais t'en donner, mais laisse-moi tranquille.» D'où l'importance, comme je le disais plus haut, de construire un espace avant que ces rêves-là puissent, peu à peu, prendre sens et devenir véritablement ce qu'ils sont: des outils de pensée.

RL: Est-ce que tu dirais qu'il faut que l'analyste rêve lui-même le rêve du patient?

VV: Exactement.

RL: Avant que le patient puisse lui-même en faire quelque chose?

VV: C'est ça, exactement. Je dirais que le patient a besoin d'être rêvé par l'analyste avant de pouvoir rêver lui-même.

RL: Alors, dans ce texte-là, tu poses l'œuvre créatrice comme un équivalent du rêve... Freud propose cette équivalence. Est-ce que l'œuvre créatrice - quand on pense à une peinture, un roman, un film — est vraiment un équivalent du rêve? Parce que l'œuvre est quand même beaucoup plus «secondarisée», non?

VV: Que ce soit un rêve, un tableau, un roman, la source (ce qui donne naissance au contenu) est toujours la même: l'inconscient. Je dirais que le rêve peut être tout aussi secondarisé qu'un roman, qu'une peinture. Au même titre qu'on trouve des rêves avec un contenu brut, on trouve des tableaux comme ceux de Francis Bacon, ou des romans comme ceux d'Elfriede Jelinek. D'ailleurs, à ce propos, Nancy Huston étudie cette question du brut, dans la littérature contemporaine, dans son essai Professeurs de désespoir. Ainsi la secondarisation se situe plus, pour moi, sur un continuum allant du plus au moins. Si on revient à Romain Gary, on retrouve dans beaucoup de ses romans des thèmes qui reviennent; un en particulier: des gens qui habitent à l'intérieur d'autres gens. Il était lui-même habité par sa mère, elle avait pris possession de sa vie, elle l'a véritablement vampirisé. Cela donne donc beaucoup de sens à ses paroles: «J'ai le sentiment d'être passé à côté de ma vie», parce que oui, en fait, il a passé à côté, sa vie ne lui appartenait pas. RL: Tu te sers de Racamier, lorsque tu dis que Gary était le rêve de sa mère. Il a fait une vie de rêve, mais ce n'était pas le sien.

VV: Exactement. C'est ce qu'il a mis en scène, c'est-à-dire qu'il était téléguidé par le fantasme de sa mère et ce que je trouve puissant, c'est qu'il ne lui suffisait pas de l'écrire, il l'a agi dans la réalité. Ainsi, en écrivant sous le pseudonyme d'Émile Ajar, il va chercher son cousin Pavlovitch et ils s'entendent pour que ce dernier personnifie Émile Ajar pour les médias. 
Chaque parole d'Émile Ajar (Pavlovitch) était téléguidée par Romain Gary. Pavlovitch devenait le pantin de Romain Gary. Ce qui l'a entre autres amené au suicide, c'est qu'à un moment donné, Pavlovitch s'est mis à vouloir parler en son nom. Gary sentait que sa "créature» lui échappait, il perdait le contrôle. Alors il s'est mis à avoir des idées paranoïdes concernant les impôts, à craindre d'être poursuivi par le fisc. L'important, je crois, c'est qu'il perdait le contrôle sur sa créature.

RL: Donc, tout son parcours est une forme de recherche identitaire?

VV: Oui, c'est comme ça que je le vois.

RL: D'ailleurs, tu parles chez lui de «roman traumatique».

VV: Oui, c'est ça. Tu demandais tantôt: «Est-ce que l'œuvre d'art peut être une façon de subjectiver?» Certainement. Mais en ce qui concerne Gary, du moins une partie importante de son œuvre, on peut y voir la mise en scène du traumatisme. Je suis en train de lire actuellement De Magritte à Bacon de Maurice Corcos. Il établit un lien entre les traumatismes infantiles de ces deux artistes et leurs toiles. L'enfant enfermé dans une mère monstrueuse chez Bacon et une mère déprimée, sans chair et sans séduction chez Magritte. On peut dire que souvent, l'œuvre, en représentant le traumatisme, pourrait conduire à la subjectivité, pourrait nourrir une pensée au même titre que le rêve. Ce n'est pas ce qui se passe. La plupart du temps, l'œuvre répète le traumatisme sans subjectivation. Et ça, Gary le disait très bien: "Ce qui m’a tenu en vie c'est les femmes et l'écriture.» Il fallait toujours qu'il publie (il a écrit 47 romans) et qu'il séduise. Le trauma sans cesse remis en scène sans élaboration.

RL: Roman désubjectivé, pourrait-on penser... Évidemment, on ne l'a pas eu en analyse, mais on pourrait penser qu'il y a eu un échec de subjectivation. C'est d'autant plus triste que j'ai l'impression que ces artistes-là aident beaucoup à l'expérience de subjectivation des autres, dans la mesure où, comme le disait Pontalis: «on n'analyse pas une œuvre, c'est l'œuvre qui nous analyse». Alors, je pense que Gary a aidé ses lecteurs à se trouver eux-mêmes mais lui, on n'en est pas certain. D'ailleurs, c'est ce qu'il dit lui-même, non? VV: C'était un de mes questionnements, je me disais: "comment quelqu'un d'aussi fin, d'aussi sensible peut, d'un autre côté, être à ce point absent à luimême?». Quand on lit La vie devant soi, c'est un chef-d'œuvre de sensibilité au niveau de la finesse et de l'expression des émotions. Et pourtant, il n'utilise pas du tout cette sensibilité pour lui-même. On peut dire que sa sensibilité n'est pas à son service, mais au service du rêve de sa mère. De même qu'on a l'impression que dans La promesse de l'aube, il saisit très bien le lien d'emprise 
avec sa mère. Il dira par exemple: «chaque année, je me rends à Londres pour m'acheter des complets, même si je déteste les complets anglais, ma mère voulait que j'en porte». Et pourtant il ne parvient pas à se l'approprier, son histoire lui demeurera à jamais étrangère. La scène finale de La vie devant soi où Momo rejoint madame Rosa dans son «trou juif»; ce trou où elle s'est réfugiée pour mourir... il n'arrive pas à la quitter malgré le corps qui se décompose et les odeurs nauséabondes qui s'en échappent. Pour moi, c'est là une représentation de la position psychique de Gary dans la vie: il a passé sa vie auprès d'une mère morte qu'il n'arrivait pas à quitter. Il la couvrait de maquillage et de parfum. À ce propos, j'ai visionné une entrevue qu'il a accordée et on voit qu'il était un véritable comédien: grande cape, grand chapeau et une voix étrange, pratiquement une voix de ventriloque. Il était madame Rosa, qu'il maquille, qu'il cache. Il était le petit Momo qui, incapable de quitter madame Rosa, devient madame Rosa. C'est une des fonctions du rêve de situer le patient dans sa réalité psychique interne.

RL: On a publié récemment chez Gallimard un petit livre, je ne sais pas si c'est sa dernière entrevue mais sûrement une des dernières qu'il ait accordées, celle qu'il avait donnée pour l'émission Rencontres à Radio-Canada, quelques mois seulement avant son suicide. Il y fait un parcours de sa vie. Et il dit bien qu'il est passé à côté. On ne sait pas s'il pense déjà au suicide à ce moment-là, mais ce sentiment d'être passé à côté de sa vie est vraiment là, c'est ce qui est d'une grande tristesse.

VV: Eh oui! Ça relativise beaucoup ce qu'est une vie réussie.

RL: Mais pour moi, aussi, ça relativise ou ça désidéalise la création au sens de créer des œuvres. Winnicott nous a bien appris qu'il y avait une différence entre vivre créativement et être un créateur. Des fois, j'ai l'impression que les analystes idéalisent beaucoup les écrivains parce qu'on se nourrit beaucoup de leurs créations. Donc, on peut idéaliser ce que cela apporte à la vie intérieure de ces gens-là, mais on voit ici que ça peut être raté pour eux... Le temps passe et je voudrais qu'on parle, si tu le veux bien, de ton propre parcours de formation à l'analyse et de ce qui a été marquant. Bien sûr, tu as fait ta formation à l'Institut psychanalytique de Montréal. Mais tu as notamment mentionné tout à l'heure tes supervisions avec André Green. Donc, après ta formation, tu as choisi d'aller faire des supervisions à l'étranger avec des analystes qui t'inspiraient particulièrement. Est-ce qu'on peut en parler un peu?

VV: Oui. En fait, je trouvais malheureux de ne pas profiter davantage de ces «maîtres». Je me suis dit que l'Europe n'était pas le bout du monde. La 
première que je suis allé voir est Joyce McDougall. Je lisais ses livres puis je trouvais cela inspirant; j'aimais sa façon de travailler, j'aimais ce qu'elle était comme personne. Alors, je lui ai écrit (c'était encore des lettres à l'époque). Je lui ai expliqué un peu où j'en étais au niveau personnel et professionnel, ce que je désirais, ce qui n'était pas très clair pour moi à ce moment-là. Je voulais une chose: établir un contact avec elle et je me disais que ça se préciserait quand je me retrouverais en sa présence. Effectivement, c'est ce qui s'est passé. Elle m’a répondu: «certainement, dites-moi à quel moment vous pensez venir ». Donc, ça a commencé comme ça, c'était en 1998 je dirais. Je l'ai vue pendant cinq, six ans... jusqu'en 2003-2004.

RL: Tu allais donc à Paris?

VV: J'allais à Paris à peu près quatre, cinq fois par année pour des périodes de sept à dix jours. J'essayais de combiner ça avec de longs week-ends... Je dois dire qu'elle était très disponible... elle me facilitait les choses.

RL: Et il y avait plusieurs rencontres de supervision pendant ces séjours?

VV: Oui. En fait, on se voyait à tous les jours et on avait souvent des entrevues doubles. Donc, pour moi, c'était un bon moment: je m'en allais là-bas, j'étais à Paris, j'étais seul, je me louais un appartement et je lisais, j'allais à mes entrevues. C'était comme une espèce de mouvement vers moi, un mouvement introspectif, très intense. Finalement, je commençais à parler de patients, et c'est peut-être là la vraie supervision, comme cela doit être fait, dans le sens où je parlais de patients mais je parlais de ce qui me questionnait moi dans mes patients... et ça a été une expérience très enrichissante pour moi, vraiment. D’ailleurs, la biographie de Joyce Mc Dougall écrite par Philippe Porret il y a quelques années a pour titre Une écoute lumineuse et c'est vraiment ça. J'ajouterais: une présence lumineuse! Quand j'ai vu le titre de ce livre, je me suis dit: c'est exactement ce que je ressentais. C'était une qualité de présence assez exceptionnelle: dès qu'on entrait dans son bureau, on se sentait comme enveloppé et la disposition des fauteuils faisait en sorte qu'on était assis très proche. Je dois dire que c'était réellement un travail à deux.

RL: Tu m'as raconté avant l'entretien quelque chose qui m'est resté, parce que pour moi ça parle de cette qualité de présence. Il s'agit de ce qui s'est passé quand tu t'étais rendu chez elle après t'être perdu dans le cimetière du Père-Lachaise.

VV: C'est cela. Je me rends au Père-Lachaise, j'ai une rencontre avec elle à seize heures, je me suis dit que j'avais le temps et que j'allais y faire un tour. Donc, je me promène dans le cimetière et à un moment donné je me dis 
qu'il faut que je parte, je m'en vais à la porte par où j'étais entré: c'est barré. Je m'en vais à une autre porte: c'était barré aussi. Là, je vois l'heure qui passe, la panique s'installe peu à peu. Finalement, je finis par sortir, je prends le métro, j'arrive tout en sueurs à mon entrevue, à bout de souffle. Je m'assois et je lui explique un peu ce qui s'est passé et elle a ces paroles: «Vous savez, c'est exactement ce qui s'est passé enfant, vous étiez pris avec vos morts et la psychanalyse a représenté, alors, une ouverture vers un ailleurs»; et c'était vrai. J'ai alors fait le lien, à treize ans, avec le malaise que je sentais et dont j'ai parlé plus haut. Je sentais alors un malaise que je n'arrivais pas à identifier, j'ai alors compris l'origine de ce malaise, j'étais pris avec mes morts... une grand-mère, un oncle et un frère. Ça a fait énormément de sens sur ce qui m'avait amené à la psychanalyse, au fond, à la pensée.

RL: Donc, elle a été très importante dans ton parcours?

VV: Oui. Au niveau personnel, bien sûr, et au niveau professionnel. J'aimais beaucoup cette façon de travailler avec les objets internes, ce qu'elle appelait la scène interne, le théâtre interne: "vous savez, les objets internes sont des personnages avec des besoins, des désirs aussi vrais que les vrais. Ils habitent notre psychisme, en prennent possession parfois et colorent toute notre vie». Je trouve, pour ma part, que ça donne une représentation du psychisme très vivante, très dynamique qui «parle» beaucoup aux patients. C'est aussi une approche que j'ai travaillée avec Jean Bégoin un peu plus tard.

RL: Si tant est qu'on devient toujours analyste, ça ne se termine pas quand on a fini notre formation, on le sait bien. Parce qu'avec McDougall, ça s'est passé après ta formation. Et tu as aussi vu André Green...

VV: C'est ça. J'ai vu Green, je suis allé dix jours à Paris, on s'est vus à tous les jours. Ça c'est autre chose, c'est différent. C'était un homme assez bourru dans un premier contact. Je lui avais écrit en lui expliquant un peu ce que je désirais: je lui présenterais du matériel, des entrevues de patients et il me dirait ce qu'il pense, les associations qui lui viennent. Je lui présenterais des patients pour lesquels j'étais en panne de pensées. Tout ça semblait correct et bien compris. Alors, j'arrive dans son bureau, je sors un dossier et je commence à lui parler d'un patient, de ses rêves. Au bout de 20 minutes, il m’arrête et me dit: «On s'en va où avec ça, là?!» Je fige et je lui réitère ma demande. Il dit: «Ça marche pas comme ça, il faut le verbatim...» «Vous voulez que j'arrive avec une entrevue?». "Oui, oui! Tout ce que vous avez dit, puis ce qu'il a dit, puis...» Moi, je n'avais pas ça du tout. Je lui dis: «écoutez, bon, je vais revenir demain puis on verra». Inutile de dire que je ne voulais pas continuer, je retournerais le lendemain et je lui dirais qu'on 
s'est mal compris. Le lendemain j'arrive, et je me retrouve devant un tout autre homme, très affable, très gentil, il me dit: «J'ai repensé au patient dont vous m'avez parlé, puis...» Finalement, on a fait nos dix jours et ce fut très enrichissant. Je dirais que c'était très scolaire, mais ça répondait à un besoin chez moi, besoin de structurer davantage ma pensée. Je dirais qu'il a remis au premier rang la question du transfert. On n'y accorde pas toujours, dans la pratique, l'importance qu'il peut avoir. Il me montrait avec justesse et pertinence que le patient, au fond, parle toujours de la relation. Dans les différents dossiers que je lui présentais, on pouvait suivre, quasi pas à pas, le cheminement et les mouvements du patient. C'était très éclairant. C'est un homme qui avait un grand sens clinique et une intuition assez impressionnante. À la fin, en se quittant, il me dit: «vous pouvez toujours revenir me voir, tant que je serai vivant, ça me fera plaisir, mais n'oubliez pas, vous devez apporter le verbatim des entrevues!!!». Il est malheureusement décédé quelques années plus tard. J'étais très content d'en avoir un peu profité.

RL: Comment vois-tu le rapport que l'analyste a ou doit avoir avec sa société de psychanalyse? Est-ce que c'est nécessaire pour un analyste d'être en contact avec celle-ci?

VV: C'est-à-dire que ce qui est nécessaire, c'est le contact avec des collègues, le contact et les échanges. C'est sûr que durant ces années-là, quand je suis allé à Paris, ça m'a nourri beaucoup. Au retour, je reprenais mon travail avec enthousiasme. Je remettais en marche mon appareil à penser. Il est essentiel de rester vivant dans le travail que l'on fait. Je crois que c'est Anzieu qui avait dit: «c'est facile de devenir analyste, mais très difficile d'y rester ». Rester un psychanalyste vivant. Quant à la Société de psychanalyse, pour différentes raisons, surtout personnelles, j'avais pris mes distances par rapport à elle, mais j'y reviens tranquillement.

RL: Est-ce que tu vois de l'espoir pour la pratique de l'analyse, avec cette espèce de morosité partagée par beaucoup d'analystes?

VV: J'ai l'impression que c'est un cliché de dire ça: c'est une des nombreuses crises que l'analyse a traversées; elle est faite pour durer, au même titre que l'art. Green me disait, à un moment donné, que ce qui se passe actuellement non seulement au niveau de la psychanalyse mais au niveau de la société, la «crise» psychanalytique n'en étant que le reflet, on va le payer très cher comme société. C'est-à-dire, cette absence de pensée. Il n'y a plus de pensée, on est dans l'acting et l'opératoire. Pour moi, le Japon est un bel exemple d'un pays qui est en train de payer très cher son développement économique qui repose sur un fonctionnement opératoire axé sur la performance. Les 
gens ne communiquent plus, s'enferment dans la solitude, on a juste à penser à ces jeunes qui passent leurs journées enfermés dans leur chambre à jouer sur l'ordinateur. Dès la maternelle, les enfants sont soumis à de fortes pressions de performance pour avoir accès aux grandes écoles plus tard, et ça continue au niveau du travail. Je pense à cet homme qui, dans un reportage, préférait aller dans les peep show plutôt que de faire l'amour avec sa femme, car c'était trop de pression: il fallait qu'il tienne compte d'elle, qu'il performe! Pour te gratifier un peu, il faut que tu ailles dans un café flatter un chat parce que tu ne trouves personne: rencontrer quelqu'un c'est te mettre dans une situation de performance! Pas surprenant, dans ces conditions, que le taux d'alcoolisme soit si élevé. Pourtant, c'est une super puissance.

La psychanalyse, on va y revenir à un moment donné; on ne peut pas continuer. On évacue tout ce qui est humain. Bon, il faut dire que je suis optimiste de nature mais...

RL: Est-ce que les analystes ont quelque chose à faire dans la société pour essayer de garder quelque chose de la pensée? Bien sûr, on voit nos patients dans nos bureaux mais...

VV: Je pense, oui. Je pense à Roussillon quand il est venu à Québec, il y a quelques années...

RL: Oui, pour le congrès de la Société canadienne de psychanalyse.

VV: Oui. Il a alors dit que le psychanalyste était vraiment le mieux équipé pour comprendre l'autisme et qu'il fallait prendre la rue, il fallait le dire, se montrer. Je regarde ce qui se passe actuellement, au procès de Magnotta; je pense qu'un analyste aurait eu beaucoup à apporter dans la compréhension de ces différents comportements. Par exemple, qu'est ce qui le motivait à porter les vêtements de sa victime? Les experts psychiatres consultés n'apportent aucune compréhension dynamique sur les motivations de cet individu. Ce qu'on en dit est vraiment minimum.

RL: C'est vrai qu'on reproche aux analystes de ne pas assez prendre la parole dans le social. On s'en est longtemps défendus en disant que la confidentialité et le transfert faisaient qu'on ne pouvait pas être des figures trop publiques, mais peut-être qu'on paye un peu pour ça?

VV: C'est ça! Je suis convaincu qu'on a quelque chose d'essentiel à apporter. La société est en mutation, les gens se questionnent, que l'on pense à la violence dans les sports, les couples, les homicides d'enfants, la procréation assistée, etc. Ce ne sont pas les thèmes qui manquent. Je pense que les gens sont avides de pensée, d'élaboration. Dans ce sens, on pourrait élargir la gamme des sujets apportés à la Table Ronde ${ }^{2}$ qui offre une structure déjà 
en place, élargir davantage son mandat qui était, si je ne me trompe pas, de démystifier la psychanalyse. Est-ce que c'est ce qui se passe actuellement? Comme tu le mentionnes, il est probable qu'on paie aujourd'hui pour notre retrait. Bien sûr, c'est beaucoup plus large que ça, mais ça reste un facteur à considérer. Il est certain qu'avec les séminaires offerts aux jeunes professionnels $^{3}$, on est sur la bonne voie. Les changements sont lents, on en sait quelque chose!

RL: Peut-être qu'on a quand même quelque chose à mettre au travail...

VV: À continuer, oui!

RL: Bien, je pense qu'on a fait le tour de nos thèmes pour cet entretien. Je te remercie beaucoup!

VV: C'est moi qui te remercie!

\section{Notes}

1. Société psychanalytique de Montréal.

2. Activité de la Société psychanalytique de Montréal, ouverte au grand public, où des invités psychanalystes et d'autres professions se rencontrent et discutent autour d'un thème.

3. Autre activité offerte depuis quelques années à la Société psychanalytique de Montréal.

\section{Références}

Vézina, V. (1985). Expérience de psychodrame analytique avec des enfants borderline. Dans Pédopsychiatrie, 20 ans à Notre-Dame. Montréal: Hôpital Notre-Dame.

Vézina, V. (1992). Plongeon ou chute? Lettre à Célina Déniger. Bulletin de la Société psychanalytique de Montréal, 4 (3), 21-22.

Vézina, V. (1995). Paradoxe, emprise et rupture. Filigrane, nº 4, 83-94.

Vézina, V. (2004). Un lion dans la jungle. Contre-transfert, traumatisme et relation objectale. Filigrane, 13 (1), 46-61.

Vézina, V. (2007). Et si ce n'était qu'un rêve. Rêve, trauma et création. Filigrane, 6 (2), 40-53

Vézina, V. (2012). Cet art qu'est la psychanalyse. Rêver des rêves inrêvés et des cris interrompus par Thomas H. Ogden. Revue canadienne de psychanalyse, 20 (2), 340-343. 